\title{
Modeling Spatial Nonstationarity via Deformable Convolutions for Deep Traffic Flow Prediction
}

\author{
Wei Zeng, Member, IEEE, Chengqiao Lin, Kang Liu, \\ Juncong Lin, Member, IEEE, Anthony K. H. Tung, Senior Member, IEEE
}

\begin{abstract}
Deep neural networks are being increasingly used for short-term traffic flow prediction, which can be generally categorized as convolutional (CNNs) or graph neural networks (GNNs). CNNs are preferable for region-wise traffic prediction by taking advantage of localized spatial correlations, whilst GNNs achieves better performance for graph-structured traffic data. When applied to region-wise traffic prediction, CNNs typically partition an underlying territory into grid-like spatial units, and employ standard convolutions to learn spatial dependence among the units. However, standard convolutions with fixed geometric structures cannot fully model the nonstationary characteristics of local traffic flows. To overcome the deficiency, we introduce deformable convolution that augments the spatial sampling locations with additional offsets, to enhance the modeling capability of spatial nonstationarity. On this basis, we design a deep deformable convolutional residual network, namely DeFlow-Net, that can effectively model global spatial dependence, local spatial nonstationarity, and temporal periodicity of traffic flows. Furthermore, to better fit with convolutions, we suggest to first aggregate traffic flows according to pre-conceived regions or self-organized regions based on traffic flows, then dispose to sequentially organized raster images for network input. Extensive experiments on real-world traffic flows demonstrate that DeFlow-Net outperforms GNNs and existing CNNs using standard convolutions, and spatial partition by pre-conceived regions or self-organized regions further enhances the performance. We also demonstrate the advantage of DeFlow-Net in maintaining spatial autocorrelation, and reveal the impacts of partition shapes and scales on deep traffic flow prediction.
\end{abstract}

Index Terms-Traffic flow prediction, spatial nonstationarity, deformable convolution, deep learning

\section{INTRODUCTION}

$\mathrm{T}$ RAFFIC flow prediction should yield accurate projections on the expected traffic conditions, in order to support intelligent transportation systems [36]. Numerous data-driven approaches, such as auto regressive integrated moving average (ARIMA) and its variants (e.g., [28], [39]) that take advantages of repeating occurrences in temporal historical traffic data, have been developed for traffic flow prediction. However, it is a challenging task for conventional approaches to model the complex non-linear spatial and temporal patterns of traffic flows. Recently, research focus has shifted towards utilizing deep neural networks (DNNs) for traffic flow prediction. Many DNN-based solutions have been developed, including residual convolution neural networks (CNNs) that are preferable for region-wise traffic flow prediction, and graph neural networks (GNNs) for graph-structured traffic data, e.g., flow volumes on road and subway networks. Studies have revealed superior performances of DNNs than conventional approaches.

Specifically, CNN approaches typically partition an underlying territory into grid-like regions, and aggregate inand out-flows in each region. In this way, traffic flows are represented in raster image format, which is consumable by

- W. Zeng and K. Liu are with Shenzhen Institute of Advanced Technology, Chinese Academy of Sciences. e-mail: $\{$ wei.zeng, kang.liu\}@siat.ac.cn.

- C. Lin and J. Lin (corresponding author) are with Xiamen University, China. E-mail: \{linchengqiao, jclin\}@xmu.edu.cn.

- A.K.H. Tung is with School of Computing, National University of Singapore.E-mail: atung@comp.nus.edu.sg.

Manuscript received $x x x x, 202 x$; revised $x x x x, 202 x$.

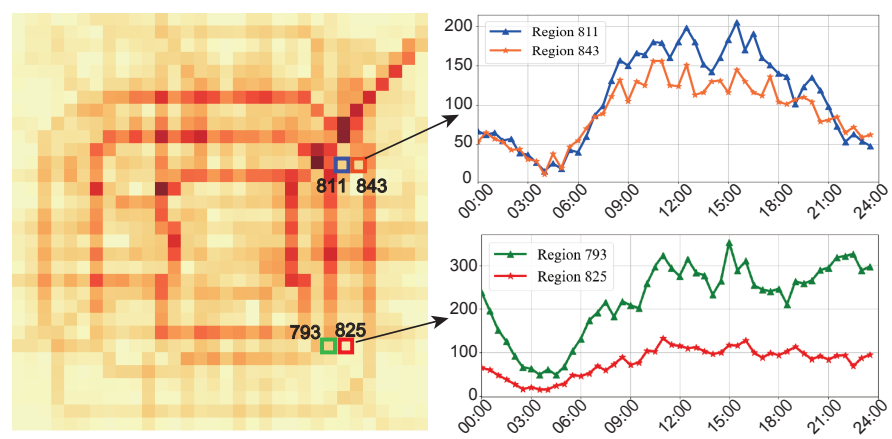

Fig. 1. Traffic flows intrinsically feature locally spatial nonstationarity between sets of neighboring regions. Traffic flows of regions $811 \& 843$ show similar temporal variations (right-top), whilst those of regions 793 $\& 825$ are distinct (right-bottom).

convolutions. Next, standard convolutions are employed to learn the spatial dependence of traffic flows between pairs of locations. Standard convolution has shown to be effective, since traffic flows observed at a spatial unit are dependent on traffic flows at nearby spatial units, which is called spatial dependence [35]. In a context of urban environments, human mobility flows are highly associated with the functionality of a place [45], [48], [16]. For example, two residences may show similar periodic patterns, e.g., large out-flows in the morning and in-flows in the evening on weekdays. In contrast, daily pendulum movements can be observed between residential and official places [49].

Besides spatial dependence, traffic flows also feature locally spatial nonstationarity that depicts varying relationships between some sets of variables over space [7]. Fig. 1 (left) 
presents traffic flows in Beijing under a $32 \times 32$ grid divison. Two sets of neighboring regions are selected: 1) regions 811 $\& 843$, and 2) regions $793 \& 825$. Temporal variations of averaged traffic flows of these regions are presented in Fig. 1 (right). Here, regions 811 \& 843 show similar flow dynamics, whilst those of regions 793 \& 825 are different. Standard convolution with a fixed geometric structure (e.g., a $3 \times 3$ kernel) cannot capture the varying relationships between the two sets of regions, not to mention if we consider sets of over two regions. As such, existing convolution-based approaches are incompetent to model spatial nonstationarity of traffic flows.

This paper tackles the problems with a feasible solution from two perspectives. First, we observe that most of existing convolution-based approaches for traffic flow prediction partition the underlying territory into grid regions. The partition scheme however increases spatial nonstationarity, and consequently harms prediction performance of the network. To mitigate the issue, we suggest to aggregate traffic flows according to pre-conceived regions (e.g., traffic analysis zones (TAZs) or functional regions), or self-organized regions based on traffic flows. The traffic flows aggregated in irregular regions are passed through a rasterization process and disposed to raster images. Second, we propose to advocate the use of deformable convolution [13], which has shown to be effective for many applications, such as semantic image segmentation [9] and image deblurring [47]. Specifically, deformable convolution augments the spatial sampling locations with additional offsets, and learns the offsets from the spatial distributions of traffic flows. In this way, deformable convolution not only inherits the ability of standard convolution in learning spatial dependence, but also being able to alter over space to capture the nonstationary characteristics of traffic flows.

On these basis, we design DeFlow-Net, which is a deformable convolutional residual network for accurate shortterm traffic flow prediction. Besides deformable convolutions for modeling spatial dependence and nonstationarity, DeFlow-Net also incorporates a temporal dependency module to learn temporal patterns of traffic flows from weekly trend, daily periodicity, and hourly closeness components. The temporal feature components are fused together to predict the future trend. We conduct extensive experiments on real-world traffic flows in Beijing, New York City, and Shenzhen. The results demonstrate that DeFlow-Net outperforms existing CNN and GNN methods for region-wise traffic flow prediction, and ablated techniques with standard and atrous convolutions, in terms of both prediction accuracy and the ability to maintain spatial autocorrelation. The study further reveals that spatial partition by pre-conceived or self-organized regions also contributes to performance improvements.

The main contributions of this work include:

- We design DeFlow-Net, a deep deformable convolutional residual network that takes advantages of deformable convolutions to model both spatial dependence and nonstationarity (Sect. 3.2). To the best of our knowledge, it is the first effort that attempts to model both spatial dependence and nonstatinarity in a deep-learning-based model for traffic prediction.

- We evaluate the effectiveness of DeFlow-Net on real- world traffic flows in three cities, and compare with baseline models and ablated techniques. The comparison results indicate our proposed model outperforms existing methods in terms of both prediction accuracy (Sect. 4.3) and the ability to preserve spatial autocorrelation (Sect. 4.4).

- We conduct local indicators of spatial autocorrelation (LISA) analysis [1], and reveal the impacts of partition shapes and scales on predictions (Sect. 4.5). The study confirms the effectiveness of integrating pre-conceived or self-organized regions (Sect. 3.1), and also the effectiveness of conventional spatial autocorrelation analysis (Sect. 4.4), when preparing network inputs.

TABLE 1

Meanings of all notations.

\begin{tabular}{|c|l|}
\hline SYMBOL & DESCRIPTION \\
\hline \hline $\mathcal{M} ; m$ & all movements; a movement. \\
\hline $\mathcal{T} ; t$ & all time slots; a time slot. \\
\hline $\mathcal{R} ; r$ & all regions; a region. \\
\hline$X_{\mathcal{R}, t} ;$ & in-/out-flow of regions $\mathcal{R}$ at time slot $t ;$ \\
$x_{r, t}$ & in-/out-flow of a region $r$ at time slot $t$. \\
\hline \hline$G ; g$ & grid map; a grid. \\
\hline$X_{G, t} ;$ & in-/out-flow of grid map $G$ at time slot $t ;$ \\
$x_{g, t}$ & in-/out-flow of a grid $g$ at time slot $t$. \\
\hline$Y_{G, t ;} ;$ & predicted in-/out-flow of grid map $G$ at time slot $t ;$ \\
$y_{g, t}$ & predicted in-/out-flow of a grid $g$ at time slot $t$. \\
\hline
\end{tabular}

\section{Problem Definition}

This section introduces fundamental concepts of our work. Table 1 lists down the notations used throughout the paper. The input data in our problem is a set of movements $\mathcal{M}$ that denotes the mobility of multiple moving objects (e.g., mobile users and taxi cabs) throughout a time period $\mathcal{T}$. We divide $\mathcal{T}$ into equal-time slots to analyze the movements in time-series discretely, i.e., $\mathcal{T}:=\left\{t_{1}, \cdots, t_{n}\right\}$.

Definition 1 (Movement). A movement $m$ is a continuously measured trajectory of a moving object during a time period $\mathcal{T}$, which is defined by a set of spatiotemporal records $\bigcup_{t \in \mathcal{T}}<t, l_{t}>$, where $l_{t}$ represents the position of $m$ at time $t$. We denote all movements of multiple moving objects as $\mathcal{M}$.

Definition 2 (Region). We partition an underlying territory into a set of regions $\mathcal{R}$ that are non-overlapping and fillup the territory. Each region $r$ can be 1) a grid partitioned based on the longitude and latitude, 2) a pre-conceived region, e.g., a functional zone isolated by road network, or a TAZ constructed by census block information for tabulating traffic-related data, or 3) a self-organized region based on interaction of traffic flows (e.g., [3], [4], [5], [6]) or similarity of human activities (e.g., [45], [46]). Pre-conceived and self-organized regions are typically in irregular shapes.

From the movements $\mathcal{M}$, we compute inflow $x_{r, t}^{I}$ and outflow $x_{r, t}^{O}$ per time slot $t$ for each region $r \in \mathcal{R}$.

Definition 3 (Inflow \& Outflow). We define the inflow $x_{r, t}^{I}$ of a region $r$ at time slot $t$ as the number of moving 
objects who are not in $r$ at time slot $t-1$ and appear in $r$ at time slot $t$,i.e.,

$$
x_{r, t}^{I}=\left|\left\{m \in \mathcal{M} \mid m . l_{t-1} \notin r \wedge m . l_{t} \in r\right\}\right|,
$$

where $|\cdot|$ denotes the cardinality of the set. Similarly, we can compute the outflow $x_{r, t}^{O}$ as

$$
x_{r, t}^{O}=\left|\left\{m \in \mathcal{M} \mid m . l_{t-1} \in r \wedge m . l_{t} \notin r\right\}\right| .
$$

For simplicity, we omit the notations $I / O$ in the following. The simplified symbols represent either inflow or outflow, unless specified. We compute in/out traffic flows for all regions $r \in \mathcal{R}$ at all time slots $t \in \mathcal{T}$, yielding a series of traffic flows $\left\{X_{\mathcal{R}, t} \mid t \in \mathcal{T}\right\}$.

Problem Definition (Short-Term Traffic Flow Prediction). Consider a set of movements $\mathcal{M}$ in the time duration of $\mathcal{T}$, a set of regions $\mathcal{R}$, and a series of derived traffic flows $\left\{X_{\mathcal{R}, t} \mid t \in \mathcal{T}\right\}$, our goal is to predict unobserved traffic flows $Y_{\mathcal{R}, t_{n+1}}$ for $\mathcal{R}$ at time slot $t_{n+1}$. Accuracy is of primary concern for short-term traffic flow prediction, i.e., output predictions shall be close to ground truths. In addition, this work also considers spatial autocorrelation as a key performance indicator, as spatial units of locally high nonstationarity are more likely to produce high prediction errors [50].

\section{Methodology}

We propose to tackle the problem from two perspectives. First, we introduce a preprocessing procedure that integrates pre-conceived or self-organized regions when preparing network consumable inputs (Sect. 3.1). Next, we present DeFlow-Net, a deep deformable convolutional residual neural network built upon deformable convolutions (Sect. 3.2). The training scheme for DeFlow-Net is described in Sect. 3.3.

\subsection{Data Processing}

As discussed in Definition 2, this work considers both grid partitions based on the longitude and latitude, and partitions by irregular pre-conceived regions such as TAZs or self-organized regions by traffic flows. The alternative partition approaches can generate different traffic flows, as statistical measurements are subject to the boundaries of spatial units, i.e., the modifiable areal unit problem (MAUP) [18], [31]. In addition, studies have shown that deep neural networks generally suffer from the adversarial perturbation problem [29], [55]. As such, the network predictions of traffic flows can be significantly different, even if the input difference is marginal. However, most of existing convolutionbased traffic prediction methods only explore grid partitions based on the longitude and latitude, e.g., [51], [43], whilst neglect partitions by pre-conceived or self-organized regions. This work examines the impacts of different partition methods on convolution-based network predictions.

- Grid partition: First, the longitude and latitude ranges of the studying area are identified. Next, the studying area is divided into grids of proper size. For example, TaxiBJ dataset partitions Beijing into $32 \times 32$ grids, BikeNYC dataset partitions NYC into $8 \times 16$ grids, and TaxiSZ dataset partitions Shenzhen into $50 \times 25$, $100 \times 50$, and $200 \times 100$ grids.

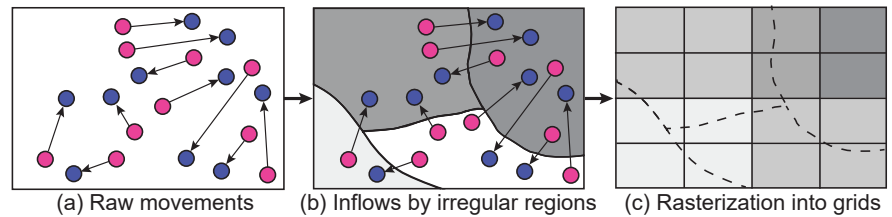

Fig. 2. A general pipeline of creating gridded traffic flows as network inputs: Raw movements (a) are counted as inflows based on irregular regions (b), and further rasterized into a grid map (c).

- Pre-conceived regions: The partition scheme integrates some subjective background knowledge to specify a set of regions. A typical example is traffic analysis zones (TAZs) constructed by census block information for tabulating traffic-related data. TAZs are used, for instance, in constructing origin-destination matrices, a classical tool of transportation engineering for describing traffic flows. Pre-conceived regions can also be incorporated to prepare traffic flows for network prediction, e.g., [50].

- Self-organized regions: There are however certain limitations for pre-conceived regions: 1) the regions are typically formed in a long time ago that may not accord with traffic flows; and 2) the number of regions are prefixed and lack of flexibility for multiscale analysis. Alternatively, one can construct selforganized regions based on traffic flows (e.g., [3], [4], [5], [6]), or human activities (e.g., [45], [46]).

To validate our model upon self-organized regions, we adopt a similarity based community division algorithm measured by traffic-flow interactions between adjacent regions. Specifically, the traffic-flow interaction $\mathrm{S}\left(r_{i}, r_{j}\right)$ is calculated as:

$$
\mathrm{S}\left(r_{i}, r_{j}\right)=\frac{2 \times\left(x_{r_{i} \rightarrow r_{j}}+x_{r_{j} \rightarrow r_{i}}\right)}{x_{r_{i}}^{I}+x_{r_{i}}^{O}+x_{r_{j}}^{I}+r_{r_{j}}^{O}}
$$

where $x_{r_{i} \rightarrow r_{j}}$ denotes the number of all-time flows from region $r_{i}$ to region $r_{j}$, and $x_{r_{i}}^{I}$ denotes the number of all-time inflow for region $r_{i}$. Note that $r_{i}$ and $r_{j}$ must meet the condition of being adjacent. Next, we merge the adjacent regions $r_{i}$ and $r_{j}$ if $\mathrm{S}\left(r_{i}, r_{j}\right)$ exceeds a predefined threshold $\alpha$. A large $\alpha$ will yield more number of regions than a small $\alpha$. The process is repeated iteratively until no more regions can be merged.

Using pre-conceived or self-organized regions in irregular shapes, the generated in/out traffic flows $X_{\mathcal{R}, t}$ show non-grid-like topology. However, a convolution operation needs inputs to be in raster-image format which can by processed by the receptive field. As such, we first convert $X_{\mathcal{R}, t}$ into a raster image $X_{G, t} \in \mathbb{R}^{i \times j}$. The process is denoted as rasterization, as illustrated in Fig. 2.

Definition 4 (Rasterization). We divide the underlying territory into a grid map $G$ of size $i \times j$ (e.g., $4 \times 4$ as in Fig. 2). Each grid $g \in G$ can intersect with arbitrary number of irregular regions $\left\{r_{k}\right\}_{k=1}^{n}$. We calculate the in/out traffic flow for each grid $g$ at time slot $t$ as:

$$
x_{g, t}=\sum_{k=1}^{n} x_{r_{k}, t} \times \frac{S\left(r_{k} \cap g\right)}{S\left(r_{k}\right)},
$$



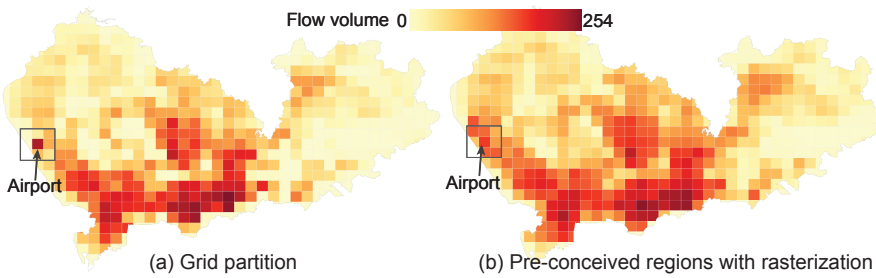

Fig. 3. Comparison of traffic flows in Shenzhen by grid partition based on the longitude and latitude (a), and by pre-conceived regions with rasterization followed (b). Both raster images are with size of $50 \times 25$.

where $S(\cdot)$ stands for the area of a region, and $r_{k} \cap g$ indicates the intersection between $r_{k}$ and $g$.

In this way, we construct a raster image $X_{G, t} \in \mathbb{R}^{i \times j}$ that represents the in/out traffic flows of the grid map $G$. We perform the rasterization process for all time slots $t \in \mathcal{T}$, yielding a series of raster images $\left\{X_{G, t} \in \mathbb{R}^{i \times j} \mid t \in \mathcal{T}\right\}$. Fig. 3 presents a comparison of traffic flows in Shenzhen by grid partition of a $50 \times 25$ grid map (a) and pre-conceived regions of 491 TAZs in Shenzhen (b). There are noticeable differences between the two raster images. Especially in the airport area, grid partition produces an extreme high volume for the grid in the center, whilst the grid has similar flow volume with those surrounding grids in the preconceived regions. The reason is that the TAZ where locates the airport is big, and the traffic flows are averaged by grids intersecting with the TAZ. The input differences have a significant impact on prediction accuracy of the outputs, as we will show in the experiment.

\subsection{DeFlow-Net}

Overview. We design DeFlow-Net that takes a series of raster images $\left\{X_{G, t} \in \mathbb{R}^{i \times j} \mid t \in \mathcal{T}\right\}$ as inputs, and predicts traffic flows $Y_{G, t_{n+1}}$ for the grid map $G$ at time slot $t_{n+1}$. The predicted traffic flow $Y_{G, t_{n+1}}$ can be expressed as:

$$
Y_{G, t_{n+1}}=\mathcal{F}_{\theta}\left(\left\{X_{G, t_{1}}, X_{G, t_{2}}, \cdots, X_{G, t_{n}}\right\}\right),
$$

where $\mathcal{F}_{\theta}(\cdot)$ represents the proposed DeFlow-Net model, while $\theta$ are the learnable parameters. Fig. 4 presents the overall framework of DeFlow-Net, which is composed of the following modules:

- Temporal Dependency Module (Sect. 3.2.1). The module models the periodic patterns of traffic flows using temporal components of weekly trend, daily periodicity, and hourly closeness.

- Deformable Convolution Module (Sect. 3.2.2). The module learns spatial dependence and nonstationarity using deformable convolutions, and deepens the convolutional layers using residual unit.

- Fusion and Activation Module (Sect. 3.2.3). Last, DeFlow-Net fuses the three temporal components to model spatial-temporal correlation, and activates the final prediction.

\subsubsection{Temporal Dependency Module}

Traffic flows in historical data feature periodic patterns. Most of the studies on short-term traffic flow predictions, including deep-learning-based approaches (e.g., [22], [51]), make use of this property. Following previous conventions,
DeFlow-Net employs three components of weekly trend that associates distant time slots in one-week, daily periodicity that associates near time slots in one-day, and hourly closeness that associates recent time slots in a few hours, to model temporal dependency.

Inputs for weekly trend component are a subsequence of raster images from the previous week. We can define the weekly trend component as $\left[X_{G, t-\Delta w \cdot p_{w}}, X_{G, t-(\Delta w-1) \cdot p_{w}}, \ldots, X_{G, t-p_{w}}\right]$, where $\Delta w$ represents length of the raster image subsequence from the previous weeks, and $p_{w}$ is fixed to one-week length, i.e., $p_{w}=48 \times 7$. Similarly, we define daily trend component as $\left[X_{G, t-\Delta d \cdot p_{d}}, X_{G, t-(\Delta d-1) \cdot p_{d}}, \ldots, X_{G, t-p_{d}}\right]$, where $\Delta d$ represents length of the raster image subsequence from the previous days, and $p_{d}$ is fixed to one-day length, i.e., $p_{d}=48$. In the closeness component, we select the previous $\Delta c$ time slots of traffic flows to infer the next time slot. Hence, the closeness component is defined as $\left[X_{G, t-\Delta c}, X_{G, t-(\Delta c-1)}, \ldots, X_{G, t-1}\right]$.

\subsubsection{Deformable Convolution Module}

Traffic flows that measure continuous movements across spatial units are by nature spatially dependent. For instance, regions with similar functionality [45] or POI distributions [48] are likely to show similar traffic flows as well. We utilize a Deformable Convolution Module to model spatial dependence among traffic flows.

Inputs of the module are trend, periodicity, and closeness components from the Temporal Dependency Module. We first concatenate the sequential raster images in each component as one tensor. Taking the closeness component for example, we concatenate both inflow and outflow raster images $\left[X_{G, t-\Delta c}, X_{G, t-(\Delta c-1)}, \ldots, X_{G, t-1}\right]$ together, yielding $X_{G, c}^{(1)} \in \mathcal{R}^{2 \Delta c \times i \times j}$ (notation (1) indicates input for the first convolutional layer). $X_{G, c}^{(1)}$ is then fed into multiple convolutional layers. The transformation matrix for the $l$-th convolutional layer can be defined as:

$$
X_{G, c}^{(l+1)}=f_{c}\left(W_{G, c}^{(l)} * X_{G, c}^{(l)}+b_{G, c}^{(l)}\right),
$$

where $*$ denotes the convolution operation, while $W_{G, c}^{(l)}$ and $b_{G, c}^{(l)}$ are two sets of learnable parameters in the $k$-th convolution layer. $f_{c}(\cdot)$ is the activation function, for which we use the rectified linear unit $(\operatorname{ReLU})$, i.e., $f_{c}(z)=\max (0, z)$. To ensure the input and output have the same size, we use zero paddings for grids at the boundary.

Convolutional Layers. Convolution is applied to make use of the spatial relationship among nearby grids covered by the receptive field. Intuitively, convolutions can learn spatial dependence because traffic flows in neighboring regions can affect each other. There are three convolution alternatives (see Fig. 5) that can lead to different effects:

- Standard convolution. Consider a location $\mathbf{p}$ (red unit in the top row of Fig. 5) in $X_{G, c}^{(l+1)}$, a standard convolution can be defined as:

$$
X_{G, c}^{(l+1)}[\mathbf{p}]=\sum_{k \in \mathcal{K}} W_{G, c}^{(l)}[\mathbf{k}] * X_{G, c}^{(l)}[\mathbf{p}+\mathbf{k}]
$$



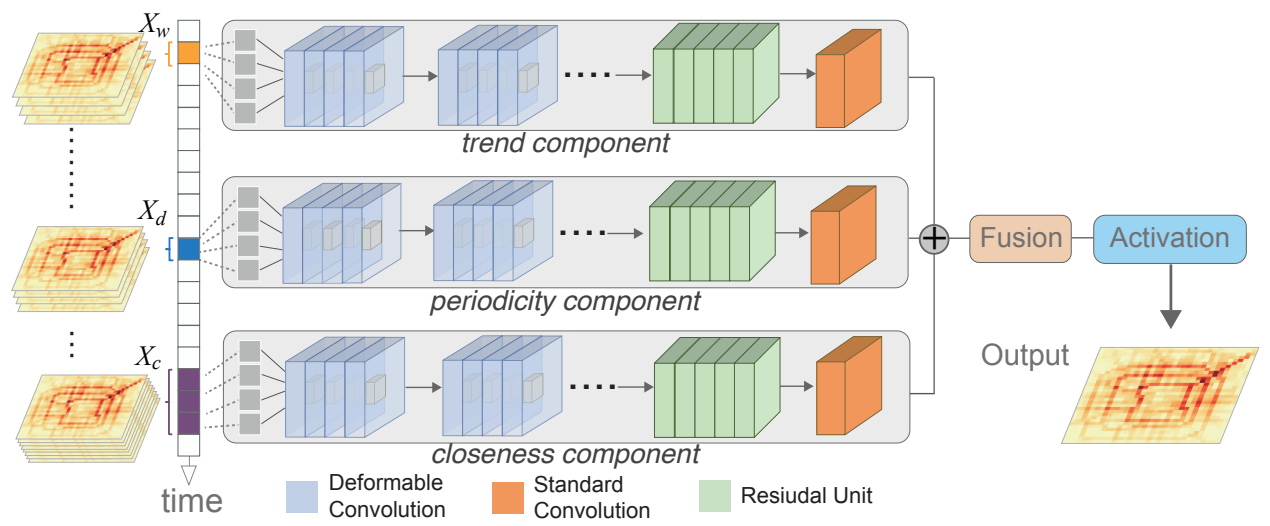

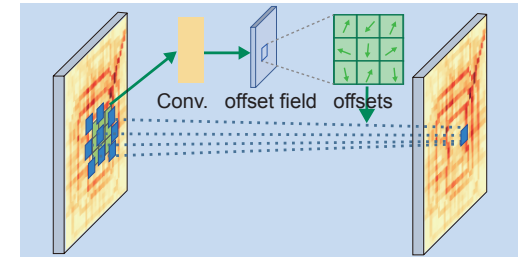

(a1) Deformable convolution

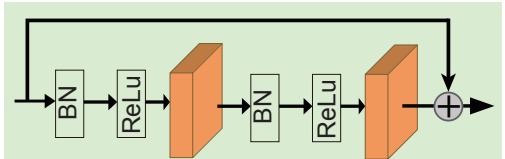

(a2) Residual unit

Fig. 4. Network architecture of our DeFlow-Net, which mainly consists of three modules: (i) a temporal dependency module including weekly trend, daily periodicity, and hourly closeness components to learn periodic patterns; (ii) a deformable convolution module taking advantage of deformable convolutions (b1) to learn spatial dependence, and residual units (b2) to increase network depth; and (iii) a fusion and activation module to fuse temporal components and activate the final prediction.

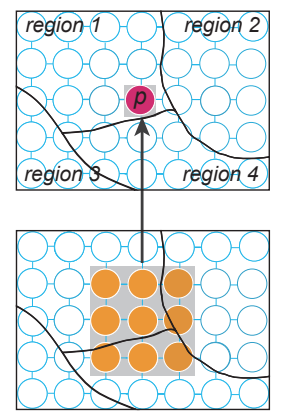

(a) Standard convolution

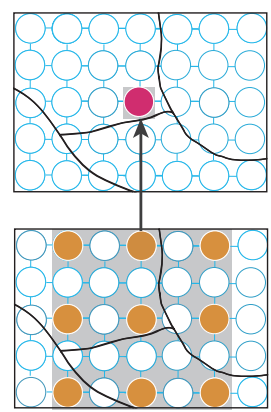

(b) Atrous convolution

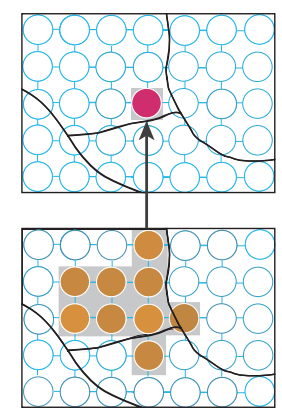

(c) Deformable convolution

Fig. 5. Illustration of the convolution alternatives. Left to right: (a) the receptive field of a standard convolution covers grids from different regions; (b) atrous convolution (atrous rate $r=2$ ) enlarges the receptive field but the grids are also from different regions; (c) deformable convolution covers neighboring grids or grids in the same region.

where $X_{G, c}^{l}[\mathbf{p}+\mathbf{k}]$ represents pixel at the position $\mathbf{p}+\mathbf{k}$ in $X_{G, c^{\prime}}^{(l)}$ and $\mathbf{k}$ is the location offset in $\mathcal{K}$. For a kernel of the size $3 \times 3, \mathcal{K}$ is defined as:

$$
\mathcal{K}=\{(-1,-1),(-1,0), \ldots,(0,1),(1,1)\} .
$$

Standard convolution is commonly adopted in many $\mathrm{CNN}$ architectures. However, standard convolution may not be optimal for learning spatial dependence in short-term traffic prediction. As shown in Fig. 5(a), the unit marked in red is in region 1 , whilst the receptive field of a standard convolution covers units distributed in 4 different regions. If the regions have different functionalities, spatial dependence of traffic flows among these units would be low, causing weak feature representation.

- Atrous convolution. Atrous convolution enlarges a convolution's field-of-view by inserting holes between pixels, to capture objects at multiple scales. Atrous convolution can be defined as:

$$
X_{G, c}^{(l+1)}[\mathbf{p}]=\sum_{k \in \mathcal{K}} W_{G, c}^{(l)}[\mathbf{k}] * X_{G, c}^{(l)}[\mathbf{p}+r \times \mathbf{k}],
$$

where $r$ is the atrous rate that specifies $r-1$ zeros along each spatial dimension. In case that $r=1$,

atrous convolution is equivalent to standard convolution. As such, atrous convolution can be regarded as a general form of standard convolution.

Atrous convolution can improve the feature capturing capability with a large atrous convolution, as shown in Fig. 5(b). However, the receptive field is still symmetric, forcing the convolutional layer to learn spatial dependence in symmetric units. In contrast, regions are typically in irregular shapes. In this sense, atrous convolution may even worsen the feature representation of spatial dependence, as it learns from distant regions [35].

- Deformable convolution. The deficiency of standard and atrous convolutions also applies to image segmentation of segmenting an image into multiple segments in regular shapes. Deformable convolution is proposed to address the deficiency, by introducing additional 2D offsets to the receptive field [13]. A deformable convolution can be expressed as:

$$
X_{G, c}^{(l+1)}[\mathbf{p}]=\sum_{k \in \mathcal{K}} W_{G, c}^{(l)}[\mathbf{k}] * X_{G, c}^{(l)}[\mathbf{p}+\mathbf{k}+\Delta \mathbf{k}],
$$

where $\Delta \mathbf{k}$ is the offset applied to the location offset k. Fig. 5(c) shows an illustration of a deformable convolution applied to traffic flows. Here, the receptive field overlaps much with region 1 , where $\mathbf{p}$ is located. The other two sampling units are outside region 1 , but they are neighbors of $\mathbf{p}$. That is, the deformable convolution effectively learns spatial dependence from neighboring units, or those located in the same region with $\mathbf{p}$.

As shown in Fig. 4(b), the offset $\Delta \mathbf{k}$ is obtained through a convolutional layer appended after a standard convolution layer. In this way, DeFlow-Net can realize the standard back-propagation for end-to-end training. It is worth noting that learnable parameters $\Delta \mathbf{k}$ are mostly fractional. We complete the convolution by bilinear interpolation, in order to obtain the values at the fractional positions.

Residual Unit. Nevertheless, one convolutional layer can only account for dependence in nearby regions, due to the limited size of kernels. To further enrich the spatial 
dependence between distant regions, DeFlow-Net utilizes multiple deformable convolutional layers. However, error back propagation becomes more difficult as the number of layers increases, causing the model degradation and vanishing gradient problems. Residual network [23] is introduced to address the problem, by utilizing shortcut connections achieved with batch normalization, ReLU activation, and standard convolution, as illustrated in Fig. 4(c). The residual unit can be expressed as:

$$
X_{G, c}^{(l+1)}=X_{G, c}^{(l)}+\mathcal{F}_{r}\left(X_{G, c}^{(l)}, W_{G, c}^{(l)}\right),
$$

where $\mathcal{F}_{r}(\cdot)$ denotes the residual function, and $W_{G, c}^{(l)}$ is the weight matrix set associated with the residual unit that needs to be learned. Note here standard convolution is adopted instead of deformable convolution to reduce computational cost. Prediction performance is not affected since spatial nonstationarity has been learned by deformable convolutional layers already.

Finally, we fuse the feature maps in each component through a standard convolution.

\subsubsection{Fusion and Activation Module}

After multiple convolutional layers and residual units in the Deformable Convolution Module, we derive $X_{G, c}^{\prime}$ for the closeness component. Similarly, we derive $X_{G, d}^{\prime}$ and $X_{G, w}^{\prime}$ for the periodicity and trend components, respectively. Next, DeFlowNet employs a fusion layer to fuse the three components together, to simultaneously model the spatial and temporal correlations. The fusion layer can be expressed as:

$$
X_{G}^{F u s i o n}=W_{c} \circ X_{G, c}^{\prime}+W_{d} \circ X_{G, d}^{\prime}+W_{w} \circ X_{G, w}^{\prime},
$$

where $W_{c}, W_{d}$, and $W_{w}$ are learnable parameters that adjust the degrees of closeness, periodicity, and trend components, respectively. We use the Hadamard product for 0 , which produces the sum of element-wise multiplication of two matrices. Last, we employ an active function to predict traffic flow $Y_{G, t_{n+1}}$ at time slot $t+1$ for the grid map $G$. The activation can be expressed as:

$$
Y_{G, t_{n+1}}=\tanh \left(X_{G}^{F u s i o n}\right),
$$

where tanh is a hyperbolic tangent that ensures the output values are between -1 and 1 .

\subsection{Training Scheme}

We normalize the training data to $[-1,1]$ using Max-Min normalization on the input datasets. Then, we denormalize the predictions and compare them with the groundtruths. We use the Adam optimizer to minimize mean squared error between the predicted traffic flow $Y_{G, t_{n+1}}$ and the groundtruth $X_{G, t_{n+1}}$ :

$$
\mathcal{L}(\theta)=\left\|X_{G, t_{n+1}}-Y_{G, t_{n+1}}\right\|_{2}^{2},
$$

where $\theta$ is learnable parameters in our model.

We implement DeFlow-Net with Keras that uses TensorFlow as the backend. DeFlow-Net can be trained in an end-to-end manner via back-propagation. All kernels of the convolutions are set to $3 \times 3$ in size. The parameters for the
TABLE 2

Statistics of the datasets used in the experiment.

\begin{tabular}{|l|c|c|c|}
\hline Dataset & TaxiBJ & BikeNYC & TaxiSZ \\
\hline \hline Data type & Taxi GPS & Bike rent & Taxi GPS \\
\hline Location & Beijing & New York & Shenzhen \\
\hline Time period (days) & 528 & 183 & 181 \\
\hline Time slot (minutes) & 30 & 60 & 30 \\
\hline Available time slots & 22,459 & 4,392 & 8,688 \\
\hline \hline Has TAZ? & No & No & 491 TAZs \\
\hline Grid map size & $32 \times 32$ & $8 \times 16$ & $\begin{array}{c}50 \times 25 \& \\
100 \times 50\end{array}$ \\
\hline
\end{tabular}

three temporal components are set as: $\Delta w=1, \Delta d=1$, and $\Delta c=3$, which empirically produces good results. The batch size during the training is set to 32 and the learning rate is set to 0.001. In addition, to obtain optimal model parameters and prevent overfitting, we perform the earlystopping strategy during training to control the number of epochs. All experiments were conducted on a server (AMD Ryzen 72700 8-Core Processor $\times 16$, NVIDIA GeForce RTX 2080 GPU) with Linux operation system.

\section{EXPERIMENT}

We conduct extensive experiments to evaluate the effectiveness of our proposed DeFlow-Net. This section presents the datasets (Sect. 4.1), baseline models and ablated techniques (Sect. 4.2), and quantitative comparison results (Sect. 4.3). In the end, we further perform spatial autocorrelation analysis (Sect. 4.4), and discuss the impacts of different partition shapes and scales (Sect. 4.5).

\subsection{DataSets}

We use three real-world datasets to evaluate the performance of our model: TaxiBJ, BikeNYC, and TaxiSZ. Statistics of the datasets are summarized in Table 2, and the details are described as follows:

- TaxiBJ: TaxiBJ records taxicab GPS data in Beijing, during four periods from 1st Jul. 2013 to 30th Oct. 2013, 1st Mar. 2014 to 30th Jun. 2014, 1st Mar. 2015 to 30th Jun. 2015, and 1st Nov. 2015 to 10 th Apr. 2016. There are in total 528-day records divided into 30-minute time slots, yielding a total of 22,459 time slots (some slots are omitted due to corrupted data). The city of Beijing is divided into a $32 \times 32$ grid map according to the longitude and latitude. The data from the last four weeks are used for testing, while the rest are used for training.

- BikeNYC: BikeNYC records bike trajectories taken from the the NYC Bike System, from 1st Apr. 2014 to 30th Sept. 2014. There are in total 183-days records divided into 60-minute tile slots, yielding a total of 4,392 time slots. BikeNYC is organized in a grid map of scale $8 \times 16$. We take the last ten days data for testing, and the rest for training.

- TaxiSZ: TaxiSZ records taxi transactions carried out by over 20k taxis during the period from 1 st Jan. 2019 to 30th Jun. 2019. Unlike publicly available TaxiBJ and BikeNYC that have been cleaned up and preprocessed, TaxiSZ contains approximately $800 \mathrm{k}$ 
raw transaction records per day, making a total of over 145 million transaction records in 181 days. The raw data include numerous corrupted or incomplete information, such as positions outside Shenzhen or missing get-on/-off times. We clean up the data by removing these records, yielding about 128 million transaction records ready for use. Next, we divide the data into 30-minute time slots, resulting in a total of 8,688 time slots. We use the data from the last two weeks for testing, and the rest for training.

Besides, we also have 491 TAZs that are designated by the transportation department in Shenzhen for tabulating traffic-related census data. The TAZ borders correspond well with recognizable physical boundaries, such as main streets and waters. In this way, the land use and activities within each TAZ are relatively homogeneous. We use the TAZ information to evaluate the benefits of partition by preconceived regions.

\subsection{Baselines and Ablations}

To evaluate the effectiveness of DeFlow-Net, we first compare with five baseline models as follows:

- HA: Historical Average (HA) uses historical average traffic flows of a given region at the corresponding time. We average traffic flows of the last three week to make the estimation.

- ARIMA: Auto-Regressive Integrated Moving Average (ARIMA) is a well-known method for predicting future trends of a time series. ARIMA has been widely used in traffic flow prediction.

- ST-ResNet [51]: ST-ResNet is one of the first convolution-based deep traffic flow prediction models. ST-ResNet can also incorporate external factors such as weather information, which is omitted in the experiment for fair comparison.

- ST-3DNet [22]: ST-3DNet exploits a specially designed 3D CNN architecture to learn spatial and temporal features in traffic flows simultaneously. It is one of the latest convolution-based deep traffic flow prediction models.

- T-GCN [53]: T-GCN learns to capture spatial dependencies from network topology with graph convolution, and temporal dependence from dynamic changes of traffic status with gated recurrent units. It was originally proposed to predict traffic data on urban road network. We model neighboring topology of the regions as a graph, and employ T-GCN to predict region-wise traffic flows.

We use the same training scheme for the baseline models, including loss function and kernel size, as those in DeFlowNet. Next, we also compare to two ablated techniques that utilize standard convolutions and atrous convolutions, instead of deformable convolutions in DeFlow-Net.

- Standard Convolution: We use a fixed kernel of $3 \times 3$ size to account for adjacency relationships. All other settings are the same with those in DeFlow-Net.

- Atrous Convolution: We use a $3 \times 3$ kernel with an atrous rate of 2 . This setting uses 9 parameters, but achieves the same field of view as a $5 \times 5$ standard convolution kernel. All other settings are the same with those in DeFlow-Net.

Last, we test the impacts of partition shapes and scales on prediction performances. We compare grid, TAZ, and self-organized partition for TaxiSZ, whilst omit TaxiBJ and BikeNYC that have been preprocessed using grid partition.

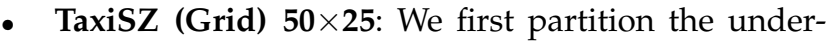
lying territory of Shenzhen into $50 \times 25$ grids based on the longitude and latitude, then aggregate in- and out-flows in each grid.

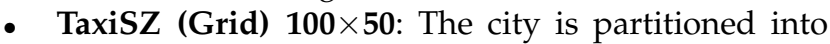
$100 \times 50$ grids for aggregation of in- and out-flows.

- TaxiSZ (TAZ) $\mathbf{5 0} \times \mathbf{2 5}$ : We first aggregate traffic flows in each of the 491 TAZs, followed by a rasterization process using $50 \times 25$ grid map.

- TaxiSZ (TAZ) $\mathbf{1 0 0} \times \mathbf{5 0}$ : The rasterization is performed upon a $100 \times 50$ grid map after aggregation.

- TaxiSZ (Self-Organized Region): We generate two sets of regions - one with 539 regions, and another one with 804 regions, by controlling the threshold as described in Equation 3. The basic units for constructing these self-organized regions are derived from a fine-grained demographic dataset with over $1 \mathrm{k}$ census blocks in Shenzhen. For each set of regions, we rasterize into $50 \times 25$ and $100 \times 50$ grid maps.

We train a DeFlow-Net model for each of these TaxiSZ variants. In the following, we abbreviate TaxiSZ (Grid) $50 \times 25$ as TaxiSZ, which is of similar size with TaxiBJ, when comparing to baseline models and ablated techniques.

\subsection{Performance Comparison}

Evaluation Metrics: To evaluate and compare the performance of different models, we first adopt root mean square error (RMSE) as in previous studies.

$$
R M S E=\sqrt{\frac{1}{N} \sum_{g=1}^{N}\left(x_{g, t_{n+1}}-y_{g, t_{n+1}}\right)^{2}},
$$

where $N$ is the number of grids, $x_{g, t_{n+1}}$ and $y_{g, t_{n+1}}$ are the ground truth and predicted traffic flow for grid $g$ at time slot $t_{n+1}$. However, RMSE measurements are unit dependent, making it unsuitable for comparison between different datasets. To address the problem, we further incorporate mean absolute scaled error (MASE) [25].

$$
M A S E=\frac{\frac{1}{N} \sum_{g=1}^{N}\left|x_{g, t_{n+1}}-y_{g, t_{n+1}}\right|}{\frac{1}{T-m} \sum_{g=1}^{N} \sum_{t=m+1}^{T}\left|x_{g, t}-x_{g, t-m}\right|},
$$

where $x_{g, t_{n+1}}$ and $y_{g, t_{n+1}}$ in the numerator are from the testing data, while $x_{g, t}$ and $x_{g, t-m}$ in the denominator are from the training data, respectively. $T$ is the total number of time slots in the training data, and $m$ is the seasonality of the time series (i.e., 48 for TaxiBJ and TaxiSZ, and 24 for BikeNYC). MASE is unit independent, allowing us to compare traffic flow predictions in different cities and at different scales. Moreover, MASE can handle actual values of zero and is not biased by very extreme values, which are problematic for mean absolute percentage error (MAPE) [25]. In general, a MASE less than 1 indicates a model is better than the naive model, and lower MASE indicates better model. 
TABLE 3

Comparison with baseline models.

\begin{tabular}{c|c|c|c|c|c|c}
\hline \multirow{2}{*}{ Model } & \multicolumn{2}{|c|}{ TaxiBJ } & \multicolumn{2}{c|}{ NYCBike } & \multicolumn{2}{c}{ TaxiSZ } \\
\cline { 2 - 7 } & RMSE & MASE & RMSE & MASE & RMSE & MASE \\
\hline HA & 52.77 & 0.605 & 10.76 & 0.230 & 12.41 & 0.409 \\
ARIMA & 28.46 & 0.672 & 9.98 & 0.245 & 11.37 & 0.437 \\
ST-ResNet & 17.34 & 0.295 & 6.48 & 0.171 & 6.54 & 0.395 \\
ST-3DNet & 17.14 & 0.292 & 5.95 & 0.168 & 5.62 & $\mathbf{0 . 2 3 4}$ \\
T-GCN & 39.68 & 0.633 & 8.78 & 0.221 & 9.36 & 0.411 \\
\hline DeFlow-Net & $\mathbf{1 5 . 9 0}$ & $\mathbf{0 . 2 7 8}$ & $\mathbf{5 . 8 5}$ & $\mathbf{0 . 1 6 5}$ & $\mathbf{5 . 3 5}$ & 0.236 \\
\hline
\end{tabular}

\subsubsection{Comparison with Baselines}

Experimental results of comparison with baseline models are shown in Table 3. The best performance for each dataset is marked in bold. Here, conventional time-series models, i.e., $H A$ and ARIMA, produce large RMSEs and MASEs. This is probably because $H A$ and ARIMA only make use of periodic patterns in temporal dimension, whilst deeplearning-based techniques further take advantages of spatial dependence. Among the baselines (ST-ResNet, ST-3DNet, and T-GCN), ST-3DNet always achieves better performances for all datasets. The result indicates that 3D convolutions can better learn spatial and temporal features, in comparison with 2D convolutions used in ST-ResNet, and graph convolutions used in T-GCN. Specifically, T-GCN achieves the worst performance among the deep learning models. A possible reason is that graph convolutions can only capture adjacent relationships among the regions, whilst neglects the spatial dependence in data with regular grid structure, e.g., traffic flows in regions.

On the other hand, our DeFlow-Net is also based on 2D convolutions, but still achieves better performances in terms of RMSE and MASE for all experimental datasets. Specifically, our model reduces RMSEs to $15.90,5.85,5.35$, and MASEs to $0.278,0.165,0.236$, for TaxiBJ, NYCBike, and TaxiSZ respectively. That is, DeFlow-Net achieves on average $4.59 \%$ RMSE and $1.91 \%$ MASE improvements, in comparison with ST-3DNet. The result confirms the effectiveness of deformable convolutions that are employed in DeFlow-Net, in modeling spatial characteristics of historical traffic flows.

\subsubsection{Comparison with Ablated Techniques}

Table 4 presents the comparison results of ablated techniques using standard and atrous convolutions. We can notice that the ablation with atrous convolutions performs better in RMSE than the one with standard convolutions on all three datasets. This is probably because atrous convolutions have a larger receptive field, allowing the ablation to better capture multi-scale spatial contextual information. Yet the improvements in terms of MASE is marginal. This indicates that the fixed geometric structure utlized by atrous convolutions, still limits its capability in modeling spatial nonstationarity of local traffic flows. Our model with deformable convolutions achieves better performances on all datasets. Notice that the improvements on different datasets are slightly different. In details, our model with deformable convolutions reduces RMSE by $7.99 \%, 5.03 \%$, $7.60 \%$, and reduces MASE by $5.76 \%, 3.51 \%, 40.25 \%$ than the ablation with atrous convolutions, for TaxiBJ, BikeNYC, and TaxiSZ, respectively. The improvements on TaxiBJ and
TABLE 4

Comparison with ablated techniques of different convolutions.

\begin{tabular}{c|c|c|c|c|c|c}
\hline \multirow{2}{*}{ Convolution } & \multicolumn{2}{|c|}{ TaxiBJ } & \multicolumn{2}{c|}{ BikeNYC } & \multicolumn{2}{c}{ TaxiSZ } \\
\cline { 2 - 7 } & RMSE & MASE & RMSE & MASE & RMSE & MASE \\
\hline Standard & 17.34 & 0.295 & 6.48 & 0.171 & 6.54 & 0.395 \\
Atrous & 17.22 & 0.295 & 6.16 & 0.175 & 5.79 & 0.477 \\
Deformable & $\mathbf{1 5 . 9 0}$ & $\mathbf{0 . 2 7 8}$ & $\mathbf{5 . 8 5}$ & $\mathbf{0 . 1 6 5}$ & $\mathbf{5 . 3 5}$ & $\mathbf{0 . 2 3 6}$ \\
\hline
\end{tabular}

TaxiSZ datasets are more significant. A possible reason is that the sizes of TaxiBJ and TaxiSZ grid maps are bigger, leaving more space for the model to learn sampling offsets.

\subsection{Spatial Autocorrelation Analysis}

Spatial autocorrelation is an important term in spatial statistics, which describes variation of a variable within geographic space. Positive spatial autocorrelation indicates the tendency for locations that are close together to have similar values. There exist many indicators for spatial autocorrelation analysis, such as Moran's I [30] and Geary's C [17]. Both metrics are one single numeric statistic for measuring global spatial autocorrelation. To quantify local effects that manifest spatial nonstationarity, this work adopts local indicators of spatial association (LISA) analyses that decompose the global Moran's I statistic to local Moran's I indices.

Definition 5 (Local Indicators of Spatial Association). Consider an in-/out-flow matrix $X_{G, t}$, we conduct LISA analysis for a local unit $x_{g_{i}, t}$ as:

$$
I\left(x_{g_{i}, t}\right)=\frac{x_{g_{i}, t}-\bar{X}_{G, t}}{S^{2}} \sum_{j=1, j \neq i}^{n} w_{i j}\left(x_{g_{j}, t}-\bar{X}_{G, t}\right)
$$

where $x_{g_{i}, t}$ indicates flow volume of grid $g_{i}$, and $x_{g_{j}, t}$ is the flow volume of grid $g_{j}$ that is one neighbor of grid $g_{i}$ at time slot $t$. Here, we choose $3 \times 3$ surrounding grids as neighbours, corresponding to the size of receptive fields. $w_{i j}$ is an element of spatial weights matrix that measures spatial connectivity between grids $g_{i}$ and $g_{j} . \bar{X}_{G, t}$ is the mean flow volume of all grids in $G$, while $S^{2}$ is the variance of flow volumes of neighboring grids.

A positive value of $I\left(x_{g_{i}, t}\right)$ indicates that grid $g_{i}$ has similarly high or low traffic volume as its neighbours, whilst a negative value indicates that $g_{i}$ is a spatial outlier. We also conduct LISA analyses for the predicted traffic flows in the same way. Fig. 6 presents Q-Q plots for LISA indicators of groundtruth (x-axis) and predicted (y-axis) traffic flows by (a) standard convolutions + grid partition, (b) deformable convolutions + grid partition, (c) standard convolutions + TAZ partition, and (d) deformable convolutions + TAZ partition. All grid maps are of the size $50 \times 25$. We can observe that grid partition produces many spatial outliers which have less than zero LISA indicators of the groundtruth traffic flows in (a) \& (b).

More importantly, we would like to examine and compare standard and deformable convolutions in terms of preserving spatial autocorrelation. To do so, we further draw a diagonal line that passes through the coordinate origin and forms the 45 degree angle with the positive direction of the $\mathrm{x}$-axis in each subfigure, as the reference. Ideally, all points shall fall along the diagonal line, indicating LISA indicators 


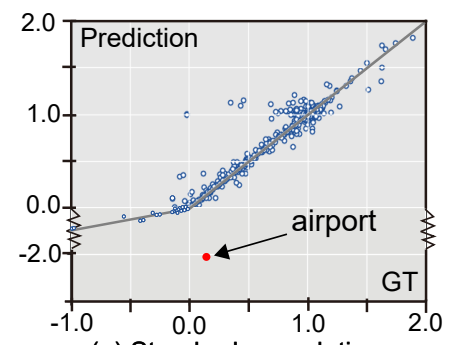

(a) Standard convolutions + Grid partition

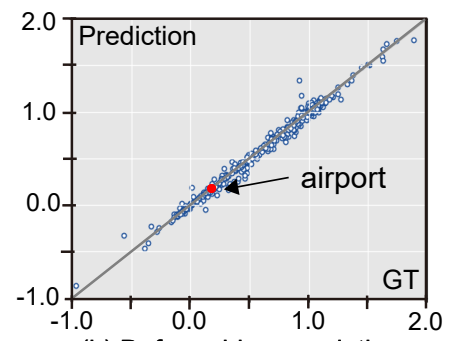

(b) Deformable convolutions + Grid partition

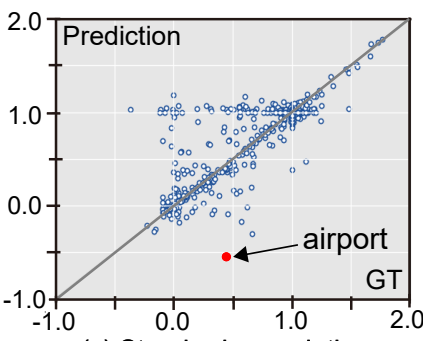

(c) Standard convolutions + TAZ partition

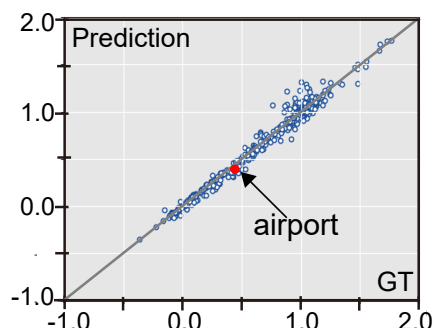

(d) Deformable convolutions + TAZ partition

Fig. 6. $Q-Q$ plots of LISA indicators of groundtruth (x-axis) and predicted (y-axis) traffic flows by different combinations of spatial partition shapes and convolution types. Deformable convolutions can better preserve spatial autocorrelation, as the points in (b) \& (d) fall close to the diagonal line, whilst many points in (a) \& (c) are far distributed from the diagonal line.

TABLE 5

Performance comparison of different partition shapes (grid vs. TAZ) and scales $(50 \times 25$ vs. $100 \times 50)$ on TaxiSZ.

\begin{tabular}{c|c|c|c|c|c}
\hline \multirow{2}{*}{ Convolution } & \multirow{2}{*}{ Metric } & \multicolumn{2}{|c|}{$50 \times 25$} & \multicolumn{2}{c}{$100 \times 50$} \\
\cline { 3 - 6 } & & Grid & TAZ & Grid & TAZ \\
\hline \multirow{2}{*}{ Standard } & RMSE & 6.54 & 6.81 & 3.09 & 2.41 \\
& MASE & 0.395 & 0.424 & 0.354 & 0.412 \\
\hline \multirow{2}{*}{ Atrous } & RMSE & 5.79 & 5.73 & 2.90 & 2.27 \\
& MASE & 0.477 & 0.461 & 0.344 & 0.422 \\
\hline \multirow{2}{*}{ Deformable } & RMSE & 5.35 & 3.09 & 2.63 & 2.12 \\
& MASE & 0.236 & 0.322 & 0.329 & 0.347 \\
\hline
\end{tabular}

of groundtruth and predicted traffic flows are the same for all grids. By comparing standard and deformable convolutions, we can notice that deformable convolutions can better preserve spatial autocorrelation, as the points in (b) \& (d) fall close to the diagonal line, whilst many points in (a) \& (c) are far distributed from the diagonal line. Taking the airport as an example, the grid is far away from the diagonal line in in (a) \& (c), whilst it is just besides the diagonal line in (b) \& (d). This indicates that the ablated techniques using standard convolutions mess up predictions for the airport and neighboring grids, which may mislead users (e.g., taxi drivers) to wrong places when picking up passengers.

\subsection{Impacts of Partition Shape and Scale}

We further evaluate the performance of DeFlow-Net upon various partition schemes. Here we divide the underlying territory into different shapes (grids $v s$. TAZs vs. selforganized regions) and scales $(50 \times 25$ vs. $100 \times 50)$. We also compare the results with ablated techniques using standard and atrous convolutions. Table 5 presents the experiment results for grid and TAZ partition, which reveal some interesting findings:

- First, our proposed DeFlow-Net outperforms the ablated techniques in all partition schemes, in terms of both RMSE and MASE. Notice that at partition scale $100 \times 50$, the improvements by DeFlow-Net is relatively small than that at scale $50 \times 25$. One possible reason is that at the finner scale, traffic flows are small for most of the grids, making spatial stationary across geographic space. In such case the benefits of deformable convolutions drop.

- Second, finer scale (i.e., $100 \times 50)$ always produces better results than coarse scale (i.e., $50 \times 25)$ in
TABLE 6

Performance comparison of self-organized partition with varying number of regions (539 vs. 804$)$ and scales $(50 \times 25$ vs. $100 \times 50)$.

\begin{tabular}{c|c|c|c|c}
\hline Num. Regions & \multicolumn{2}{|c|}{$539(\alpha=0.01)$} & \multicolumn{2}{c}{$804(\alpha=0.02)$} \\
\hline Grid map & $50 \times 25$ & $100 \times 50$ & $50 \times 25$ & $100 \times 50$ \\
\hline RMSE & 3.07 & 2.03 & 3.68 & 2.23 \\
MASE & 0.323 & 0.330 & 0.415 & 0.443 \\
\hline
\end{tabular}

terms of RMSE. The results however do not infer that finer scale is better, but simply because RMSE is a unit-dependent metric. In contrast, the unitindependent metric MASE recommends coarse scale $50 \times 25$. Future researches shall take care about the unit dependence issue.

- Last, at the same partition scale, DeFlow-Net with TAZ partition always produces better results than with grid partition in terms of RMSE, but not of MASE. Through careful investigation, we find the higher MASEs by TAZ partition mainly come from some grids at the boundary regions. The rasterization process assigns some marginal traffic flows to these grids, and eventually cause large MASEs.

Table 6 further presents the performance comparison of self-organized partition with 539 and 804 regions made by different threshold values. We also compare the results under different grid map size $(50 \times 25$ vs. $100 \times 50)$. All the results are achieved by DeFlow-Net with deformable convolutions. Here, the prediction accuracy measured by both RMSE and MASE improves when the final number of regions decreases from 804 to 539. A possible reason is that traffic flows are averaged when merging adjacent regions, i.e., spatial dependence increases as the number of regions decreases. Nevertheless, the results do not infer that less regions are better, as it would be practically more useful to predict traffic flows for fine-grained regions. Hence, we stop merging regions when the number reaches 539, which is similar to the number of regions (491) of TAZ partition. Moreover, we can notice that self-organized partition with 539 regions achieves a slightly better performance than TAZ partition with 491 regions (Table 5), even though the number of regions by self-organized partition is more. This is probably because that self-organized partition based on traffic flows can better accord with spatial autocorrelation as compared to TAZ partition. 


\section{Related Work}

This work focuses on short-term forecasting for traffic flows [36]. We group related work into three categories: Traffic flow prediction summarizes conventional and lately deep-learning-based methods for traffic flow prediction; spatial nonstationarity discusses spatial nonstationarity of traffic flows, and its impacts on deep traffic flow prediction; and deformable convolutions introduces recent developments of deformable convolution networks.

\subsection{Traffic Flow Prediction}

Traffic flow prediction can be regarded as a classic timeseries forecasting problem. As such, many conventional methods for traffic flow prediction are based on time-series models, such as autoregressive integrated moving average (ARIMA) (e.g., [28], [39]) and structural time-series model (STM) [19], which take advantage of repeating occurrences in temporal historical data to fit parametric models. An alternative approach is nonparametric regression, including $k$-nearest neighbors $(k-\mathrm{NN})$ (e.g., [8], [40]) and Bayesian network (e.g., [34]). Comparison study [33] showed that parametric models generally outperform nonparametric regressions, yet the performance of nonparametric regressions can be significantly improved by larger databases. For instance, a large value of $k$ coupled with larger databases can provide a better set of neighbors in $k$-NN models, and consequently improve the prediction accuracy.

Nevertheless, the complexity of finding neighbors increases dramatically with larger $k$ values and database sizes. Deep neural networks (DNNs) can suppress the challenge by learning hidden features in traffic flows via dedicated network architectures. As a result, DNNs are being increasingly used for traffic flow prediction. Early attempts with fully connected neural networks [24], [27] achieve superior performances than conventional approaches. Lately, CNNs (e.g., [51], [15], [52], [20]) and GNNs (e.g., [42], [54], [21], [53]), have been utilized for predicting traffic flows. CNNs model spatial dependency by decomposing the traffic network as grids, while GNNs are more appropriate for graphstructured traffic data, e.g., the road network [44].

This work focuses on predicting traffic flows in regions, for which CNNs are more frequently utilized. The experimental results (Sect. 4.3.1) have shown that both 2D and 3D CNNs outperform T-GCN using graph convolution networks. This is probably because graph convolutions can only capture adjacent relationships among the regions, but not the spatial dependence and nonstationarity properties among regions.

However, existing convolution-based methods typically adopt standard convolutions with fixed geometric structures to learn spatial dependence across the whole space. The assumption of spatial stationarity is problematic, since a global trend do not reflect the underlying data generating processes [7]. Instead, we employ deformable convolutions that are competent to model the sophisticated spatial nonstationarity in local traffic flows.

\subsection{Spatial Nonstationarity}

When modeling spatial-temporal dynamics, Anselin emphasized in his influential textbook [2] two intrinsic char- acteristics of spatial data that need to be taken into account: spatial dependence and spatial nonstationarity (or spatial heterogeneity). As Tobler's first law of geography [35] observed, "everything is related to everything else, but near things are more related than distant things", spatial dependence refers to a situation where attribute values observed at one spatial unit are dependent on neighboring values at nearby spatial units. In contrast, spatial nonstationarity points to the lack of spatial stationarity in attribute values of a particular measure across all spatial units [7].

By far, the literature of convolution-based deep traffic flow prediction concentrates on modeling spatial dependence, whilst little attention has been put on modeling spatial nonstationarity. Yao et al. [43] showed that including regions with weak correlations for a target region can hurt the prediction performance, and designed local CNNs to filter weakly correlated remote regions. Cheng et al. [12] proposed a dynamic spatio-temporal $k$-nearest neighbor model to capture the heterogeneous spatio-temporal pattern of road traffic. Their work is inspiring but differs with our approach that utilizes deformable convolutions to automatically learn spatial nonstationarity from traffic flows. Perhaps most similar to our work is DST-ICRL [15] that combines irregular convolutional residual network with long short term memory network. However, DST-ICRL needs to divide passenger flows of different traffic lines or routes into multiple channels, and utilizes irregular convolution kernels to capture the interpretable high-level dependency in each individual channel. The process is time-consuming and may damage the dependence among spatial units. Instead, our proposed DeFlow-Net reserves traffic flows of all spatial units as one single input, being able to learn spatial dependence and nonstationarity simultaneously.

\subsection{Deformable Convolution}

Convolutional layers that learn abstract feature maps using convolution kernels, are basic components in convolutional neural networks. Standard convolution kernels are defined by fixed shapes of equal width and height (e.g., $3 \times 3$ or $5 \times 5$ ), which however may lose some neighboring information as the receptive field of a standard convolution kernel only covers an area with checkerboard patterns [38]. The deficiency may impede certain prediction tasks that feature dynamic spatial correlations, such as semantic segmentation and traffic flow prediction.

Atrous convolution, also known as dilated convolution, inserts holes (i.e., trous in French) between pixels to enlarge the field of convolution kernels [11]. A well-known example is DeepLab [10] that employs Atrous Spatial Pyramid Pooling (ASPP) to capture multi-scale objects and context information by placing multiple dilated convolution layers in parallel. Although atrous convolution can enable dense feature extraction, the convolutional kernels are designed to sample the input tensors at symmetric positions, making it difficult to align key points or salient features at arbitrary positions. Therefore, atrous convolutions can still lead to the loss of total information given the discontinuous sampling [41].

To overcome the limitation, Dai et al. [13] proposed deformable convolution as a general form of atrous convolution, by learning sampling offsets dynamically from input 
tensors. Due to its generalizability, deformable convolution has been successfully applied in many computer vision applications, e.g., semantic segmentation [9], image deblurring [47], and video enhancement [14]. We introduce deformable convolutions for traffic flow prediction, and design DeFlow-Net accordingly. Experimental results demonstrate the superior performance of deformable convolutions than standard and atrous convolutions.

\section{Discussion, Conclusion, AND Future WORK}

Though being an intrinsic property, the local nonstationary characteristic of traffic flows has been seldom considered in deep traffic flow prediction. This work calls for attention to the impacts of spatial nonstationarity on convolution-based DNN models. We tackle the problem from the perspectives of network inputs and architecture.

First, we show that spatial partition schemes, including both partition shapes and scales (i.e., the MAUP [18], [31]), can affect local nonstationarity across the geographic space. Consequently, prediction performance of CNN models are different. Specifically, spatial partition according to preconceived regions (TAZs) and self-organized regions tend to relax spatial nonstationarity and increase prediction accuracy, in comparison to grid partition based on the longitude and latitude. Besides, finer-grained partition scale (TaxiSZ $100 \times 50$ ) always produces smaller absolute errors than coarse scale (TaxiSZ $50 \times 25$ ), yet the inference is not reliable if we consider unit independent metrics, e.g., MASE adopted in this work. Hence, we suggest

Insight 1: to integrate pre-conceived or self-organized regions with traffic flows when preparing network inputs, and choose appropriate partition scale according to practical requirements.

Second, realizing the impacts of spatial nonstationarity, we design DeFlow-Net (Sect. 3.2), a deep deformable convolutional residual network that incorporates deformable convolutions to enhance the capability of extracting spatial features. DeFlow-Net outperforms existing convolutionbased DNN models and ablated techniques, throughout all three real-world datasets. The advancements include both prediction accuracy in terms of RMSE and MASE, and the ability to preserve spatial autocorrelation. The results indicate that deformable convolutions can model both globally spatial dependence and locally spatial nonstationarity. As such, we recommend

Insight 2: to use deformable convolutions, instead of standard convolutions, when constructing the network architecture of convolution-based DNN models for deep traffic flow prediction.

Future work: There are several directions for future research, as follows. First, the DNN models used in this work are treated as as 'black box', and we apply 'what-if' analysis to examine the impacts of spatial nonstationarity on prediction performance. To better understand the working mechanism and improve the model, we would like to open the black box and display the internal states of the DNN models, such as to interpret the hidden unit response to feature selections [32]. Second, this work relies solely on traffic flows for prediction. Studies have shown that incorporating external factors, such as weather [37], [51] and context information [26], can improve the prediction performance. We would also like to redesign the network architecture to fuse these factors. Nevertheless, the heterogeneous data also exhibit independent spatial nonstationarity, bringing in more challenges for network prediction.

\section{ACKNOWLEDGMENT}

This work is supported partially by National Natural Science Foundation of China (61802388, 62077039, 41901391), Guangdong Basic and Applied Basic Research Foundation (2021A1515011700), and the Shenzhen Fundamental Research Program (JCYJ20190807163001783).

\section{REFERENCES}

[1] L. Anselin. Local indicators of spatial association-LISA. Geographical Analysis, 27(2):93-115, 1995. 2

[2] L. Anselin. Spatial econometrics: methods and models, volume 4. Springer Science \& Business Media, 2013. 10

[3] T. Anwar, C. Liu, L. V. Hai, M. S. Islam, and T. Sellis. Capturing the spatiotemporal evolution in road traffic networks. IEEE Transactions on Knowledge and Data Engineering, 30(8):1426 - 1439, 2018. 2,3

[4] T. Anwar, C. Liu, H. Le Vu, and C. Leckie. Spatial partitioning of large urban road networks. In Proceedings of International Conference on Extending Database Technology, pages 343-354, 2014. 2, 3

[5] T. Anwar, C. Liu, H. L. Vu, and M. S. Islam. Tracking the evolution of congestion in dynamic urban road networks. In Proceedings of the 25th ACM International on Conference on Information and Knowledge Management, page 2323-2328, New York, NY, USA, 2016. 2, 3

[6] T. Anwar, C. Liu, H. L. Vu, and C. Leckie. Partitioning road networks using density peak graphs: Efficiency vs. accuracy. Information Systems, 64:22-40, 2017. 2, 3

[7] C. Brunsdon, A. S. Fotheringham, and M. E. Charlton. Geographically weighted regression: a method for exploring spatial nonstationarity. Geographical Analysis, 28(4):281-298, 1996. 1, 10

[8] H. Chang, Y. Lee, B. Yoon, and S. Baek. Dynamic near-term traffic flow prediction: system-oriented approach based on past experiences. IET Intelligent Transport Systems, 6(3):292-305, 2012. 10

[9] F. Chen, F. Wu, J. Xu, G. Gao, Q. Ge, and X.-Y. Jing. Adaptive deformable convolutional network. Neurocomputing, 453:853-864, 2021. 2, 11

[10] L. Chen, G. Papandreou, I. Kokkinos, K. Murphy, and A. L. Yuille. DeepLab: Semantic image segmentation with deep convolutional nets, atrous convolution, and fully connected CRFs. IEEE Transactions on Pattern Analysis and Machine Intelligence, 40(4):834-848, 2018. 10

[11] L.-C. Chen, G. Papandreou, F. Schroff, and H. Adam. Rethinking atrous convolution for semantic image segmentation. arXiv preprint arXiv:1706.05587, 2017. 10

[12] S. Cheng, F. Lu, and P. Peng. Short-term traffic forecasting by mining the non-stationarity of spatiotemporal patterns. IEEE Transactions on Intelligent Transportation Systems, pages 1-19, 2020. 10

[13] J. Dai, H. Qi, Y. Xiong, Y. Li, G. Zhang, H. Hu, and Y. Wei. Deformable convolutional networks. In Proceedings of the IEEE International Conference on Computer Vision, pages 764-773, 2017. 2, 5,10

[14] J. Deng, L. Wang, S. Pu, and C. Zhuo. Spatio-temporal deformable convolution for compressed video quality enhancement. In Proceedings of the AAAI Conference on Artificial Intelligence, pages 10696-10703, 2020. 11

[15] B. Du, H. Peng, S. Wang, M. Z. A. Bhuiyan, L. Wang, Q. Gong, L. Liu, and J. Li. Deep irregular convolutional residual LSTM for urban traffic passenger flows prediction. IEEE Transactions on Intelligent Transportation Systems, 21(3):972-985, 2019. 10

[16] Z. Feng, H. Li, W. Zeng, S.-H. Yang, and H. Qu. Topology density map for urban data visualization and analysis. IEEE Transactions on Visualization and Computer Graphics, 27(2):828-838, 2021. 1 
[17] R. C. Geary. The contiguity ratio and statistical mapping. The Incorporated Statistician, 5(3):115-141, 1954. 8

[18] C. E. Gehlke and K. Biehl. Certain effects of grouping upon the size of the correlation coefficient in census tract material. Journal of the American Statistical Association, 29(185A):169-170, 1934. 3, 11

[19] B. Ghosh, B. Basu, and M. O. Mahony. Multivariate short-term traffic flow forecasting using time-series analysis. IEEE Transactions on Intelligent Transportation Systems, 10(2):246-254, 2009. 10

[20] Z. Gong, B. Du, Z. Liu, W. Zeng, P. Perez, and K. Wu. SDseq2seq : A deep learning model for bus bunching prediction based on smart card data. In Proceedings of International Conference on Computer Communications and Networks (ICCCN), pages 1-9, 2020. 10

[21] S. Guo, Y. Lin, N. Feng, C. Song, and H. Wan. Attention based spatial-temporal graph convolutional networks for traffic flow forecasting. In Proceedings of the AAAI Conference on Artificial Intelligence, volume 33, pages 922-929, 2019. 10

[22] S. Guo, Y. Lin, S. Li, Z. Chen, and H. Wan. Deep spatial-temporal $3 \mathrm{D}$ convolutional neural networks for traffic data forecasting. IEEE Transactions on Intelligent Transportation Systems, 20(10):3913-3926, 2019. 4,7

[23] K. He, X. Zhang, S. Ren, and J. Sun. Deep residual learning for image recognition. In Proceedings of the IEEE Conference on Computer Vision and Pattern Recognition, pages 770-778, 2016. 6

[24] W. Huang, G. Song, H. Hong, and K. Xie. Deep architecture for traffic flow prediction: deep belief networks with multitask learning. IEEE Transactions on Intelligent Transportation Systems, 15(5):2191-2201, 2014. 10

[25] R. J. Hyndman and A. B. Koehler. Another look at measures of forecast accuracy. International Journal of Forecasting, 22(4):679-688, 2006. 7

[26] Z. Lin, J. Feng, Z. Lu, Y. Li, and D. Jin. DeepSTN+: Contextaware spatial-temporal neural network for crowd flow prediction in metropolis. In Proceedings of the AAAI Conference on Artificial Intelligence, volume 33, pages 1020-1027, Jul. 2019. 11

[27] Y. Lv, Y. Duan, W. Kang, Z. Li, and F. Wang. Traffic flow prediction with big data: A deep learning approach. IEEE Transactions on Intelligent Transportation Systems, 16(2):865-873, 2015. 10

[28] C. K. Moorthy and B. G. Ratcliffe. Short term traffic forecasting using time series methods. Transportation Planning \& Technology, 12(1):45-56, 1988. 1, 10

[29] S. Moosavi-Dezfooli, A. Fawzi, O. Fawzi, and P. Frossard. Universal adversarial perturbations. In Proceedings of the IEEE Conference on Computer Vision and Pattern Recognition, pages 86-94, 2017. 3

[30] P. A. Moran. Notes on continuous stochastic phenomena. In Biometrika, pages 17-23, 1950. 8

[31] S. Openshaw. The Modifiable Areal Unit Problem. Geo Books, Norwick, UK, 1984. 3, 11

[32] Q. Shen, Y. Wu, Y. Jiang, W. Zeng, A. K. H. LAU, A. Vianova, and H. Qu. Visual interpretation of recurrent neural network on multidimensional time-series forecast. In Procedings of IEEE PacificVis, pages $61-70,2020.11$

[33] B. L. Smith, B. M. Williams, and R. Keith Oswald. Comparison of parametric and nonparametric models for traffic flow forecasting. Transportation Research Part C: Emerging Technologies, 10(4):303-321, 2002. 10

[34] S. Sun, C. Zhang, and G. Yu. A bayesian network approach to traffic flow forecasting. IEEE Transactions on Intelligent Transportation Systems, 7(1):124-132, 2006. 10

[35] W. R. Tobler. A computer movie simulating urban growth in the detroit region. Economic Eeography, 46(sup1):234-240, 1970. 1, 5, 10

[36] E. I. Vlahogianni, J. C. Golias, and M. G. Karlaftis. Short-term traffic forecasting: Overview of objectives and methods. Transport Reviews, 24(5):533-557, 2004. 1, 10

[37] D. Wang, W. Cao, J. Li, and J. Ye. DeepSD: Supply-demand prediction for online car-hailing services using deep neural networks. In Proceedings of the IEEE International Conference on Data Engineering, pages 243-254, April 2017. 11

[38] P. Wang, P. Chen, Y. Yuan, D. Liu, Z. Huang, X. Hou, and G. Cottrell. Understanding convolution for semantic segmentation. In Proceedings of the IEEE Winter Conference on Applications of Computer Vision, pages 1451-1460, 2018. 10

[39] B. M. Williams, P. K. Durvasula, and D. E. Brown. Urban freeway traffic flow prediction: Application of seasonal autoregressive integrated moving average and exponential smoothing models. Transportation Research Record, 1644(1):132-141, 1998. 1, 10
[40] C.-H. Wu, J.-M. Ho, and D.-T. Lee. Travel-time prediction with support vector regression. IEEE Transactions on Intelligent Transportation Systems, 5(4):276-281, 2004. 10

[41] X. Xu, X. Xiong, J. Wang, and X. Li. Deformable kernel convolutional network for video extreme super-resolution. In A. Bartoli and A. Fusiello, editors, Computer Vision - ECCV 2020 Workshops, pages 82-98, Cham, 2020. Springer International Publishing. 10

[42] S. Yan, Y. Xiong, and D. Lin. Spatial temporal graph convolutional networks for skeleton-based action recognition. In Proceedings of the AAAI Conference on Artificial Intelligence, pages $7444-7452$, 2018. 10

[43] H. Yao, F. Wu, J. Ke, X. Tang, Y. Jia, S. Lu, P. Gong, J. Ye, and Z. Li. Deep multi-view spatial-temporal network for taxi demand prediction. arXiv preprint arXiv:1802.08714, 2018. 3, 10

[44] J. Ye, J. Zhao, K. Ye, and C. Xu. How to build a graph-based deep learning architecture in traffic domain: A survey. IEEE Transactions on Intelligent Transportation Systems, pages 1-21, 2020. 10

[45] J. Yuan, Y. Zheng, and X. Xie. Discovering regions of different functions in a city using human mobility and POIs. In Proceedings of the SIGKDD Conference on Knowledge Discovery and Data Mining, pages 186-194. ACM, 2012. 1, 2, 3, 4

[46] N. J. Yuan, Y. Zheng, X. Xie, Y. Wang, K. Zheng, and H. Xiong. Discovering urban functional zones using latent activity trajectories. IEEE Transactions on Knowledge and Data Engineering, 27(3):712-725, 2015. 2, 3

[47] Y. Yuan, W. Su, and D. Ma. Efficient dynamic scene deblurring using spatially variant deconvolution network with optical flow guided training. In Proceedings of the IEEE Conference on Computer Vision and Pattern Recognition, pages 3552-3561, 2020. 2, 11

[48] W. Zeng, C.-W. Fu, S. Müller Arisona, S. Schubiger, R. Burkhard, and K.-L. Ma. Visualizing the relationship between human mobility and points-of-interest. IEEE Transactions on Intelligent Transportation Systems, 18(8):2271-2284, 2017. 1, 4

[49] W. Zeng, C. W. Fu, S. Müller Arisona, A. Erath, and H. Ou. Visualizing waypoints-constrained origin-destination patterns for massive transportation data. Computer Graphics Forum, 35(8):95 107, 2016. 1

[50] W. Zeng, C. Lin, J. Lin, J. Jiang, J. Xia, C. Turkay, and W. Chen Revisiting the modifiable areal unit problem in deep traffic prediction with visual analytics. IEEE Transactions on Visualization and Computer Graphics, 27(2):839-848, 2021. 3

[51] J. Zhang, Y. Zheng, and D. Qi. Deep spatio-temporal residual networks for citywide crowd flows prediction. In Proceedings of the AAAI Conference on Artificial Intelligence, pages 1655-1661, 2017. 3, $4,7,10,11$

[52] J. Zhang, Y. Zheng, J. Sun, and D. Qi. Flow prediction in spatiotemporal networks based on multitask deep learning. IEEE Transactions on Knowledge and Data Engineering, 32(3):468-478, 2019. 10

[53] L. Zhao, Y. Song, C. Zhang, Y. Liu, P. Wang, T. Lin, M. Deng, and H. Li. T-GCN: A temporal graph convolutional network for traffic prediction. IEEE Transactions on Intelligent Transportation Systems, 21(9):3848 - 3858, 2019. 7, 10

[54] C. Zheng, X. Fan, C. Wang, and J. Qi. GMAN: A graph multiattention network for traffic prediction. In Proceedings of the AAAI Conference on Artificial Intelligence, pages 1234-1241, 2020. 10

[55] S. Zheng, Y. Song, T. Leung, and I. Goodfellow. Improving the robustness of deep neural networks via stability training. In Proceedings of the IEEE Conference on Computer Vision and Pattern Recognition, pages 4480-4488, 2016. 3

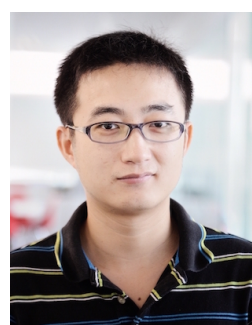

Wei Zeng is currently an associate professor at Shenzhen Institute of Advanced Technology, Chinese Academy of Sciences. He received both his B.E. and Ph.D. in computer science from Nanyang Technological University. Before joining SIAT, he worked as a senior researcher at Future Cities Laboratory, ETH Zurich. His current research interests include geospatial data analysis and visualization, $A R / V R$, and urban computing. 


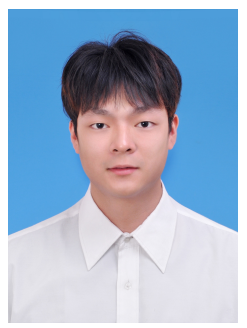

Chengqiao Lin is currently a master student in School of Informatics, Xiamen University. His research interests include spatiotemporal data mining, intelligent transportation systems and urban computing.

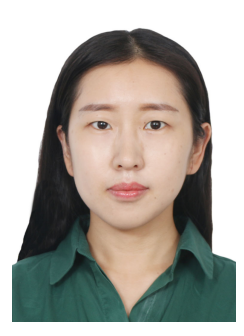

Kang Liu is currently an associate professor at Shenzhen Institute of Advanced Technology, Chinese Academy of Sciences. She obtained her Ph.D. degree in Institute of Geographic Sciences and Natural Resources Research (IGSNRR), Chinese Academy of Sciences (CAS) in 2018. Her research interests focus on geospatial data intelligence and urban computing, and application fields of intelligent transportation, public health, and urban planning.

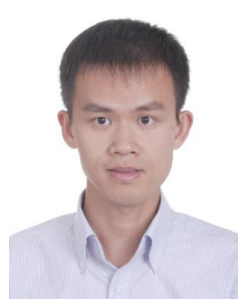

Juncong Lin is currently a full professor in School of Informatics, Xiamen University, leading the Graphics and Virtual Reality Laboratory. $\mathrm{He}$ received his B.S. and Ph.D Degree both from Zhejiang Univesity in 2003 and 2008 respectively. Before joining Xiamen University, he has worked in CUHK, NTU as a postdoc researcher. His research interests include data analysis, educational visualiazation, creativity support tools, and geometry process.

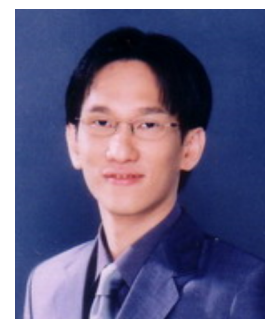

Anthony K. H. Tung is currently a Professor in the Department of Computer Science, National University of Singapore (NUS). He received both his B.Sc. and M.Sc. in computer sciences from the National University of Singapore in 1997 and 1998 respectively. In 2001, he received the Ph.D. in computer sciences from Simon Fraser University (SFU). Dr Anthony Tung main research areas are on searching, mining and visualizing complex data. More recently, he also looks into the creation of innovative big data applications over the data processing techniques that he had developed over the past 18 years. Anthony is also the deputy director of NUS NCript research center (https://ncript.comp.nus.edu.sg/). 